\title{
Sédiments et paléolimnologie d'un réservoir : le lac de Pareloup (Aveyron, France). Premiers résultats
}

\author{
T.E. Rhodes 1 \\ R. Le Cohu ${ }^{2}$
}

Mots clés : Paléolimnologie, réservoir, pigments, bacillariophyceae.

Les résultats des premières études sur les diatomées et les pigments des sédiments d'un réservoir mesotrophe sont analysés. La proportion des deux especes de diatomées les plus fréquentes Asterionella formosa et Melosira italica est inversée vers 1981-1982, années où le lac semble avoir subi une "poussée * d'eutrophisation. Cependant, aucun changement dans les concentrations en pigments n'a été détecté. Les teneurs élevées en pigments autour de l'année 1975 ne semble pas devoir être imputées à une augmentation de la production algale ; elles semblent plutôt associées à un abaissement du niveau du lac.

Sediments and paleolimnology in the Pareloup reservoir (Aveyron, France). Preliminary report.

Keywords : Paleolimnology, reservoir, pigment analysis, bacillariophyceae.

The first analysis of sedimentary pigments and sedimentary diatoms from a mesotrophic reservoir are described. Relative proportions of the two most common diatoms, Asterionella formosa et Melosira italica are reversed in assemblages from 1981-1982, two years when the lake was unusually eutrophic. However, no change in pigment concentrations appears at this level. Elevated pigment concentrations in deposits from the mid -1970's are ascribed to the effects of lower water levels during this period rather than to an actual increase in productivity.

\section{Introduction}

Le lac de Pareloup, situé à $800 \mathrm{~m}$ d'altitude au sudouest du Massif Central, a été mis en eau il y a 35 ans pour la production d'électricité et la fourniture d'eau potable. C'est un réservoir de type mésotrophe subissant chaque année un marnage de l'ordre de 7 à $8 \mathrm{~m}$. Depuis 1983, il est caractérisé par un important pic printanier de diatomées qui s'amorce dès la fin du mois de février et décline à la fin juin. Ce pic printanier est dû essentiellement au développement d'Asterionella formosa Hassal (jusqu'à $90 \%$ des cellules comptées) et à un degré moindre à Melosira italica (Ehr.) Kütz. Outre ces deux especes, Melosira ambigua (Grun.) O. Müll., Fragilaria crotonensis

1. Department of botany and plant pathology. University of Maine, Orono, ME 04469 U.S.A.

2. Laboratoire d'Hydrobiologie, U.A. 695 CNRS, Université P. Sabatier, 118, route de Narbonne, 31062 Toulouse Cedex. France. Adresse des demandes de tiré à part.
Kitton, Cyclotella comta (Ehr.) Kütz. et Cyclotella stelligera $\mathrm{Cl}$. u. Grun. apportent une contribution non négligeable au peuplement. Le déclin des dia. tomées coïncide avec l'établissement de la stratification thermique qui se maintient pendant trois mois. Les eaux au-dessous de $20 \mathrm{~m}$ de profondeur et les sédiments superficiels sont généralement anoxiques d'août à octobre.

Dans le cadre des études entreprises sur ce lac depuis 1983, une approche de la paléolimnologie nous a paru nécessaire afin d'avoir une idée des possibles variations de l'état trophique du lac et des fluctuations de la production algale.

\section{Méthodes}

D'avril à juin 1986, des prélèvements de sédiments ont été effectués périodiquement à l'aide d'une 
drague Eckman ; des carottes de 10 à $18 \mathrm{~cm}$ de longueur ont été extraites par succion à l'aide d'un tube en plastique (Ludlam 1982). Quelques échantillons de sédiments plus profonds ont été recueillis avec un carottier. La plupart des prélèvements ont été effectués dans le bassin principal (A) à $32 \mathrm{~m}$ de profondeur, les autres (B, C, D) dans 3 autres sites localisés dans les anses. Les carottes étaient coupées sur place en tranches de $1 \mathrm{~cm}$ d'épaisseur placées au réfrigérateur dès le retour au laboratoire. Les sédiments provenant du bassin principal (A) ont été datés par comptage de varves.

Le dénombrement des diatomées a été réalisé à partir de sédiments non traités; les résultats sont exprimés en pourcentage par rapport au peuplement global de diatomées.

L'extraction des pigments a été réalisée sur sédiments humides; des échantillons de $34 \mathrm{mg}$ d'une couche de carotte étaient malaxés dans $30 \mathrm{ml}$ d'acétone ou d'éthanol à $90 \%$. Le tout était laissé à l'obscurité pendant $24 \mathrm{~h}$ et agité de temps en temps (Gorham 1960, Wetzel 1970). L'analyse spectrophotométrique était effectuée $4 \mathrm{~h}$ après la dernière agitation afin d'eviter toute centrifugation ou filtration. Les concentrations en chlorophylle a et phéopiments ont été calculées à partir des équations de Marker $\&$ al. (1980). Les 2 solvants donnaient des résultats différents quand les analyses étaient répétées sur des échantillons supposés identiques. Cependant, en fonction de la profondeur, l'allure des courbes restaient la même. L'erreur standard sur 6 replicatas était raisonnablement basse (chlorophylle a\# $8 \%$; phéopigments \# 1,8\%).

\section{Résultats et discussion}

\subsection{Structure des sédiments}

Nous nous baserons essentiellement sur les résultats obtenus au site A dans le bassin principal ( $32 \mathrm{~m}$ de profondeur). Les sédiments sont constitués de gyt tja à structure fine. Le contenu en eau est élevé, $83 \%$ à $8 \mathrm{~cm}$ de profondeur (moins de $68 \%$ dans les anses, B, C et D). Les poches de gaz sont fréquentes. La perte au feu est du méme ordre dans les 4 sites prospectés, entre 14 et $18 \%$, les sites A et B étant les plus riches en matières organiques.
Chaque varve montre une alternance de couches brunes et rougeâtres, ce qui a permis de les dater (Simola \& al. 1984) et de remonter jusqu'en 1961 ; elles s'accumulent en moyenne sur $11 \mathrm{~mm}$ chaque année (fig. 1). Toutefois, après 1975 , il faut remarquer une diminution de l'épaisseur des dépôts annuels, c'est-à-dire environ 10 ans après la mise en eau. Les couches brunes représentent les sédiments superficiels à l'époque où le lac est bien stratifié et désoxygéné en profondeur (août et septembre); elles correspondent à la sédimentation des diatomées du pic printanier (un épais tapis floconneux jaunattre était observé à la surface des sédiments fin juin 1986). Les couches rouges, plus épaisses (fig. 1), sont constituées par tous les dépôts durant la longue période où la colonne d'eau est entièrement oxygénée ; elles contiennent, contrairement aux premières, beaucoup de frustules brisés et de dépóts allochtones. Il faut d'ailleurs remarquer que les couches brunes recèlent, par unité de poids, $43 \%$ de valves de plus que les couches rouges.

\subsection{Distribution des diatomées dans les sédiments}

Des frustules, en particulier de Melosira italica et de Melosira ambigua, possédant encore des chloroplastes ont été détectés d'un bout à l'autre des carottes, soit jusqu'à $29 \mathrm{~cm}$ de profondeur. Pour distinguer cellules vivantes et cellules non viables, le critère de Stockner et Lund (1970), chloroplaste intact. ne semble pas suffisant, comme l'ont démontré récemment Sicko-Goad et al. (1986). Ces derniers ont pu réaliser des cultures de Melosira granulata (Ehr.) Ralfs à partir de filaments prélevés dans des sédiments anoxiques à $20 \mathrm{~cm}$ de profondeur; les restes de chloroplastes dans les cellules ne permettaient pas " a priori " de déceler une vie potentielle. Au cours de cette étude exploratoire, nous avons donc préféré globaliser les cellules sans essayer de différencier cellules vivantes et cellules mortes, ceci afin d'éviter toute interprétation erronée.

Dans les carottes étudiées, Melosira italica, Melosira ambigua, Asterionella formosa et Fragilaria crotonensis sont les taxons les mieux représentés. Les trois premières espèces contribuent pour plus de $90 \%$ aux assemblages de diatomées. Le G. Melosira (fig. 2) est généralement majoritaire, exception faite des années 1979, 1980 et 1981. A moins de changements importants dans la composition du peuplement diatomique, cette dominance ne reflète pas ce 


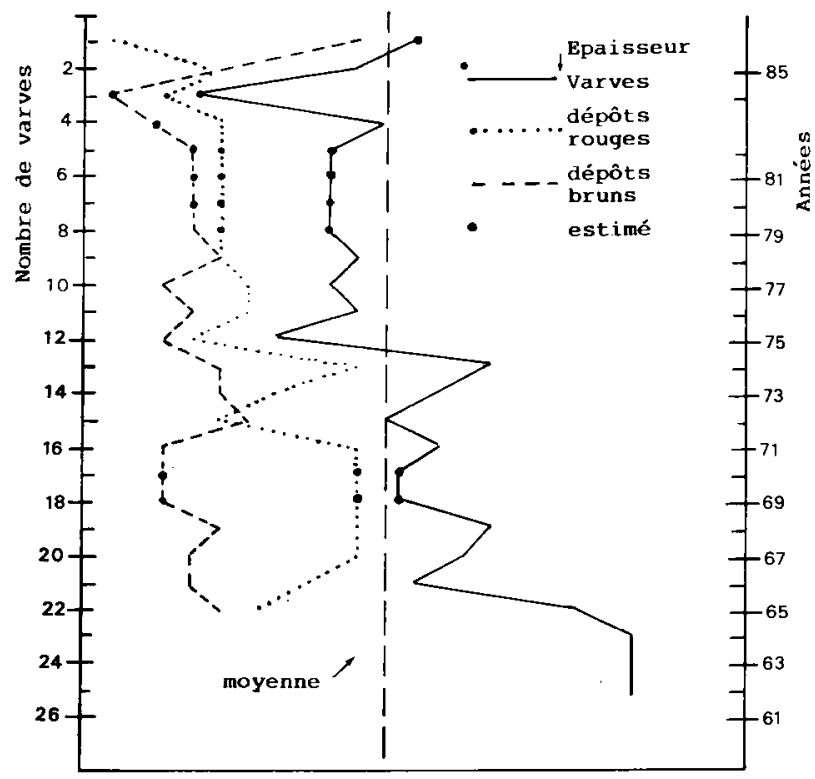

Epaisseur des couches, en mm

Fig. 1. Evolution de l'épaisseur des varves en fonction de leur nombre et de la datation ; l'épaisseur des deux types de dépôts (rouges et bruns) constituant les varves est également indiquée.

qui est observé dans le lac ; en effet, en 1981 et 1982 (Lair, comm. pers.) et depuis 1983 (Jrad, comm. pers.), la part d'Asterionella formosa est largement prépondérante au sein des diatomées planctoniques. Ces premières analyses sont en contradiction avec celles de Stockner \& Lund (1970); ces deux auteurs observaient une grande similitude entre dominance en eau libre et dans les sédiments. Les résultats obtenus dans le lac de Pareloup sont difficilement explicables dans l'immédiat. Quelques hypothèses, sous toute réserve, peuvent être avancées: fragilité des valves d'Asterionella formosa (à la fín du printemps, on observe une nette diminution de taille et une faible silicification des frustules) ; transport hors du lac, une partie des dépôts de la sédimentation printanière étant peut-être remise en suspension au moment de la période de circulation, les frustules de Melosira plus lourds, plus solides restant en place. Il est difficile d'envisager une croissance importante des deux espèces de Melosira à la surface des sédiments, même si une croissance hétérotrophe est possible (Mc Lean \& al. 1981).

\subsection{Distribution des pigments dans les sédiments}

Le graphique $n^{\circ} 3$ illustre les résultats obtenus au site $A$ à partir de deux carottes, distantes tout au plus de 3 à $4 \mathrm{~m}$. Les couches en chlorophylle a 


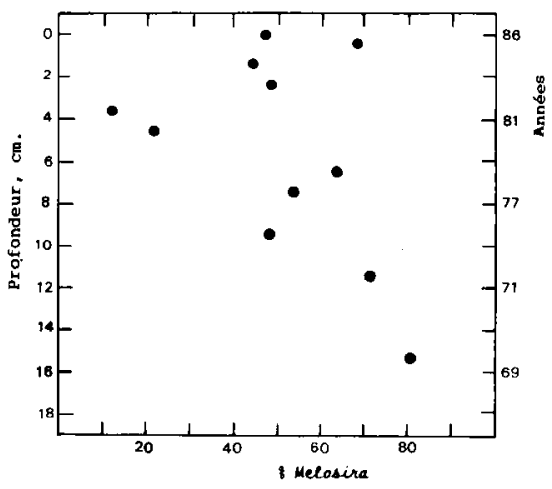

Fig. 2. Variation du pourcentage de We/ssila part rapport au nombre total de diatomées comptées en fonction de la profondeur et de la datation. L'échantillon au niveau 0 correspond à la couche floconneuse jaunâtre observée a la surface des sédiments en juin 1986.

et phéopigments sont pratiquement parallèles ; les apports d'origine allochtone restent donc à peu près constants. Même si on considère que les valeurs obtenues sont seulement indicatives, les teneurs en pigments sont élevées, avec un maximum vers $10 \mathrm{~cm}$ de profondeur, aux environs de 1975. Généralement, on admet qu'une augmentation des concentrations en pigments exprime un accroissement de la biomasse algale déposée (Wetzel 1970, Frey 1974). Au cours d'investigations portant sur des lacs italiens, Guilizoni \& al (1983) ont également mis en évidence une corrélation entre production primaire actuelle et concentrations en pigments dans les sédiments. Le pic enregistré vers $10 \mathrm{~cm}$ de profondeur traduirait donc une augmentation de la production algale, l'élévation de la teneur en chlorophylle a reflétant la présence d'algues vivantes en quantité plus importantes qu'aux autres périodes. Ce maximum ne semble pas être dû à un enrichissement momentané du lac en substances nutritives grâce à des apports exceptionnels par lessivage des sols. En effet, il n'y a jamais accumulation de sédiments lit toraux ; par exemple, en été, les matières organiques représentent seulement $4,9 \%$ du poids des sédiments. Par contre, il faut remarquer que ce pic à $10 \mathrm{~cm}$ coïncide avec un niveau anormalement bas du lac (Documents fournis par E.D.F.). II n'y aurait pas eu accroissement de la production algale mais simplement concentration de la production habituelle dans un volume d'eau moindre, donc sédimentation sur une plus petite surface. Corrélativement au bas niveau du lac, l'effluent a eu un débit nettement plus faible que d'habitude, moins d'algues ont été entraînées hors du lac. Whiteside (1983) envisage ces deux dernières possibilités comme explication possible des variations du carbone organique dans les sédiments.

Les années 1981 et 1982 ont été marquées par le développement, pendant l'été et l'automne, de fleurs d'eau à Cyanophycées, suggérant une eutrophisation accélérée du réservoir (Lair, comm. pers.). L'analyse des pigments n'en rend pas compte. Quand elles sont senescentes, il est connu que les cyanobactéries à pseudovacuoles gazeuses ont tendance, par temps calme, à s'accumuler au voisinage de la surface. Dans le cas présent, elles ont pu soit être entrainées en grande partie hors du lac soit se décomposer sur place, les produits de la minéralisation ayant été recyclés lors de la longue période d'isothermie.

\section{Conclusion}

Ces premières analyses illustrent deux difficultés dans l'utilisation des pigments pour déterminer la production algale dans un passé récent. En effet, l'interprétation peut conduire à déduire soit une apparente augmentation soit une apparente stabilité des nutriments alors que la réalité est toute autre. De plus, comme nous l'avons déjà signalé pour les Cyanophycées, les concentrations en pigments reflètent sans doute aussi très imparfaitement la biomasse produite par Asterionella formosa chaque année : l'analyse des « assemblages " de diatomées dans les sédiments l'a d'ailleurs déjà montré puisque le $\mathrm{G}$. Melosira prédomine généralement, notamment les dernières années. On peut donc envisager une dégradation rapide des pigments chez Asterionella for mosa, celle-ci, plus légère, sédimentant plus lentement que le G. Melosira (dans les pièges à sédiments à $15 \mathrm{et} 20 \mathrm{~m}$ de profondeur, de nombreuses cellules de cette espèce montrent déjà des chloroplastes très dégradés, obs. pers.). De plus, les frustules d'Asterionella formosa sont fragiles (surtout à la fin du pic 


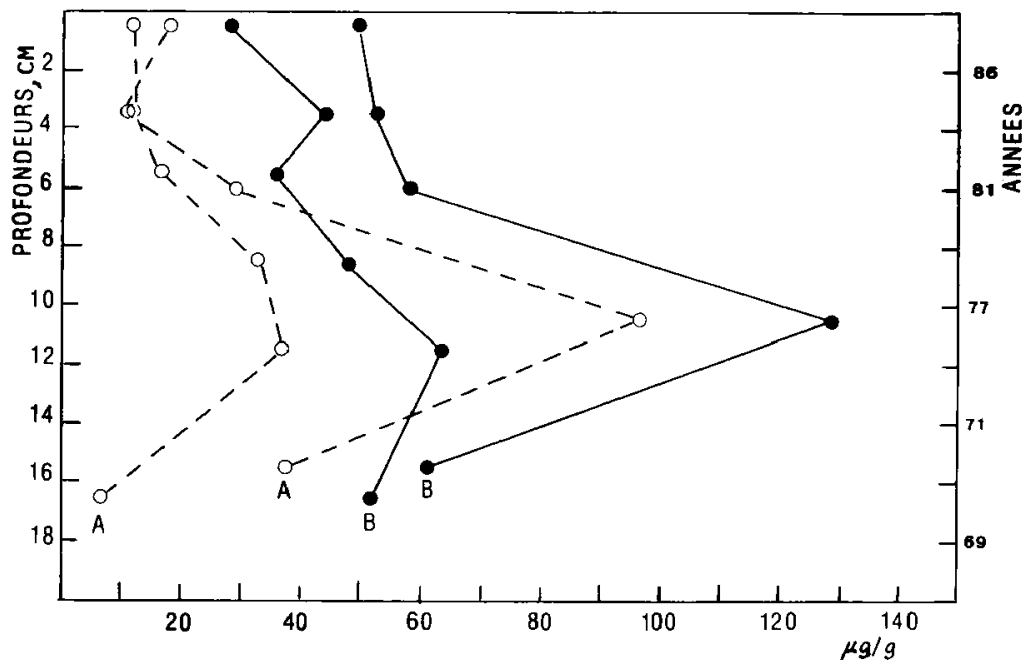

Fig. 3. Variations des concentrations (mg/g) de la chlorophylle a - . e et des phéopigments deur et de la datation - Résultats obtenus au site A sur 2 carottes. Extraction à l'acétone.

printanier) et se brisent facilement. Par ailleurs, il faut signaler, dans ce réservoir, la présence d'un inoculum potentiel de la cyanophycée Mycorcystis aeruginosa. Des cultures réalisées à partir des sédiments superficiels ont permis d'obtenir une croissance rapide de l'espèce précitée. Un tel processus de réensemensement de la colonne d'eau à partir de colonies de Mycrocystis subsistant à la surface des sédiments a d'ailleurs déjà été montré (Preston \& al. 1980).

\section{Travaux cités}

Frey (D.G.). 1974. - Paleolimnology. Mitt. Intermat. Verein. Limnol, $20: 95-123$.

Gorham (E.). 1960. - Chlorophyll derivatives in surface muds from the English Lakes. Limnol. Oceanogr., $5: 29.33$.

Guilizzoni (P.), Bonomi (G.), Galanti (G.) \& Ruggiu (D.) 1983 . Relationship between sedimentary pigments and primary pro duction : evidence from core analysis of twelve Italian lakes. Hydrobiologia, 103 : 103.106.
Ludlam (S.D.). 1982. - Use and limitations of sedimentary pigments as indicators of past lake productivity. Water Res. Research Ctr., U iv, Massachusetts, Amherst, U.S.A. $\mathbf{N}^{\circ} 127$; $63 \mathrm{p}$.

Marker (A.F.H.). Crowther (C.A.) \& Gunn (R.J.M.). 1980. Methanol and acetone as solvents for estimating chlorophyll a and phaeopigments by spectrophotometry. Arch. Hydrobiol. Beih. Ergebn. Limnol., $14: 52-69$.

Mc Lean (R.O.), Corrigan (J.) \& Webster (J.). 1981. - Heterotrophic nutrition in Melosira nummuloides, a possible role in affecting distribution in the Clyde estuary. Br. phycol. $t, 16: 95-106$.

Preston (T.), Stewart (W.D.P.) \& Reynolds (C.S.). 1980 - Bloomforming Cyanobacterium Mycrocystis ae ruginosa overwinters on sediment surface. Nature, $228,5789: 365-367$.

Sicko-Goad (L.), Stoermer (E.F.) \& Fahnenstiel (G.). 1986. Rejuvenation of Melosira grarulata (Bacillariophyceae) resting cells from the anoxic sediments of Douglas Lake. Michigan. I Light microscopy and ${ }^{14} \mathrm{C}$ uptake. J. Phycol., $22: 22.28$.

Simola (H.), Huttunen (P.) \& Merilaïnen (J.). 1984: - Varve dated eutrophication history of a small lake. Verh. Internat. Verein. Limnol., 103 : 1404-1408.

Stockner (J.G.) \& Lund (J.W.G.). 1970. - Live algae in post-glacial lake deposits. Limnol. Oceanogr, $15: 41-48$.

Wetzel (R.G.). 1970. - Recent and postglacial production rates of a marl lake. Limnol. Oceanogr., 15: 491-503.

Whiteside (M.C.). 1983. - The mythical concept of eutrophication. Hydrobiologia, 103 : 107-112. 